\title{
A economia metropolitana e os seus impactos regionais em Portugal: uma análise espacial exploratória de Lisboa, Porto e Coimbra
}

\author{
Metropolitan economy and \\ regional impacts in Portugal: an \\ exploratory spatial analysis of \\ Lisbon, Porto and Coimbra
}

\section{Iván G. Peyré Tartaruga}

Professor e investigador

Centro de Estudos de Geografia e Ordenamento do Território (CECOT), Faculdade de Letras,

Universidade do Porto, Portugal

Rede Observatório das Metrópoles (INCT/CNPq),

Núcleo Porto Alegre, Brasil

itartaruga@letras.up.pt 


\section{Resumo}

Este artigo analisa os impactos regionais das economias metropolitanas em Portugal focando as aglomerações de Lisboa, Porto e Coimbra. Teoricamente, o estudo faz uso de uma abordagem baseada nos eixos de desenvolvimento das cidades - econômico, institucional, das interações sociais e político - e do conceito de causação circular e cumulativa. Utilizando uma técnica de análise exploratória de dados espaciais (AEDE) - a estatística I de Moran - conseguiu-se estabelecer os diferentes padrões espaciais da variável produtividade. Concluiu-se que, de maneira geral, no território português ocorrem relações espaciais entre as regiões. Assim, pôde-se determinar aglomerações de baixo desempenho econômico no interior do país. No que tange às metrópoles, Porto e Coimbra não apresentaram relacionamentos espaciais importantes com seus respectivos entornos regionais. Em Lisboa revelou-se um aglomerado com desempenho positivo no seu interior, portanto, sem impactos na sua vizinhança regional. Daí decorre a conclusão da baixa estruturação econômica regional no país.

Palavras-chave: economia metropolitana, impacto regional, análise espacial, Portugal.

\section{Abstract}

This article analyses the regional impacts of the metropolitan economies of Portugal, therefore, focusing the agglomerations of Lisbon, Porto and Coimbra. Theoretically, the study used the keys of development of cities approach-economic, institutional, social interactions and political - and the concept of circular and cumulative causation. We operate with an exploratory spatial data analysis (ESDA) - Moran's I statistics - and can establish the different spatial patterns of productivity variable. We conclude that in the Portuguese territory there are spatial relations among regions. Then, we determinate agglomerations of low economic performance in the inner country. In the case of metropolises, Porto and Coimbra do not present important spatial relationships with their surrounding regions. In Lisbon observed a positive performance in an inner agglomeration, hence, without impacts on its regional neighbourhood. In conclusion, there is a low structuration of regional economy in the country.

Keywords: metropolitan economy, regional impact, spatial analysis, Portugal.

Journal of Economic Literature (JEL): R12, O18, O31. 


\section{Introdução}

As ciências em geral necessitam de dados quantitativos para seus estudos e de métodos científicos para sua análise. Nas ciências sociais, da mesma forma, aparece essa necessidade que se soma a outra: a adequação dos métodos e, consequentemente, dos dados às teorias pertinentes. As técnicas quantitativas devem responder às perguntas provenientes das teorias explicativas, que tem seu fundamento na realidade social. As ciências regionais preocupadas com a localização dos fenômenos que aborda, precisam utilizar informações referenciadas espacial e temporalmente. Uso este que tem um aumento exponencial em razão da disponibilidade de dados espaciais e da proliferação de métodos para sua análise, que se refletem, por exemplo, nos programas computacionais vinculados sistemas de informações geográficas (SIC).

O principal objetivo deste artigo é experimentar o uso de uma metodologia espacial para responder a alguns questionamentos no campo da geografia econômica. Assim, o estudo busca analisar os principais centros urbanos de Portugal, nos seus aspectos econômicos, com a finalidade de verificar seus impactos regionais em termos de desenvolvimento econômico. Tal fim tem por base o conceito de causação circular e cumulativa, derivado das teorias de desenvolvimento econômico (Scott e Storper, 2003). Para isso, utilizou-se uma técnica de análise exploratória de dados espaciais (AEDE), que verifica a influência de uma região sobre seu entorno com respeito a uma variável quantitativa, no caso deste trabalho, a produtividade.

$\mathrm{O}$ texto está dividido da seguinte forma. Na segunda seção, se estabelece o quadro teórico que orienta este artigo, e que diz, de um lado, a respeito das transformações das cidades e das regiões e, de outro, aos seus impactos regionais. $\mathrm{Na}$ terceira parte, apresentam-se a metodologia e os dados utilizados. A quarta seção revela os resultados das análises realizadas por meio de uma descrição regional do país seguida da análise espacial exploratória. A última seção finaliza com as conclusões do estudo.

\section{Cidades, regiões e seus impactos regionais}

Há muitas décadas que as ciências sociais têm interesse em encontrar explicações para o crescimento e as mudanças que ocorreram nas cidades e regiões. Entre as mais recentes e mais convincentes estão aquelas que propõem visões abrangentes em que os aspectos sociais, econômicos e ambientais são contemplados (Storper, 
2010). Assume-se um quadro teórico que estabelece a centralidade e a especificidade dos espaços urbanos e principalmente os metropolitanos. Portanto, distinguindo os fenômenos urbanos de outras dimensões sociais e que, ao mesmo tempo, reconhece a grande variedade de gêneros de cidades (Scott e Storper, 2015).

Com ênfase na sócio-economia, Storper (2013) apresenta uma interessante perspectiva para a compreensão do desenvolvimento das cidades a partir de quatro eixos integrados: econômico, institucional, das interações sociais e político. Quanto ao contexto econômico, ressalta o papel dos espaços urbanos para a geração, a manutenção e a qualificação dos empregos e para o estabelecimento das especializações produtivas que, ao longo do tempo, podem promover diversificações posteriores na produção e o surgimento de processos de inovação. O segundo eixo é o contexto institucional, formado pelas instituições governamentais formais e informais. As formais - locais, regionais e nacionais - representam a complexa rede de agentes políticos com poder de decisão relevante sobre o desenvolvimento ou a estagnação das regiões. Já as informais, conforme a abordagem institucionalista (Bathelt e Glückler, 2014), são retratadas pelas convenções e normas sociais definidas pelos diversos grupamentos humanos em relação às comunidades na escala local dos micro grupos em bairros, vilas, etc., atuando em redes interpessoais informais complexas, e à sociedade na escala de maior dimensão dos macro grupos como a nação, operando de forma mais transparente e em anonimato (Rodríguez-Pose e Storper, 2006). Por sua vez, o terceiro eixo ressalta a importância das interações sociais para a economia urbana. Estas relações locais formam a base relacional e cognitiva para o desenvolvimento tecnológico (inovações) que, também, interagem reciprocamente com a globalização (Bathelt, Malmberg e Maskell, 2004). Sobretudo, refletem-se nos contatos face a face entre os diferentes atores das cidades como cientistas, empresários, gestores públicos, etc. (Storper e Venables, 2004). O último eixo evidencia o contexto político, os aspectos normativos e decisórios das cidades e do desenvolvimento, em direção a uma maior eficiência da gestão e a uma maior justiça, considerando-se interações econômicas justas e consequências positivas para a coletividade (Storper, 2011).

Deve-se frisar que, como se verá em seguida na seção metodológica, este estudo abordou, principalmente, o eixo econômico e, indiretamente, o institucional e o socio-interacional. Eixos da abordagem do quarteto interpretativo do desenvolvimento das cidades. Enquanto, no primeiro foi aplicado o método quantitativo principal desta análise, nos dois últimos foi utilizada uma contextualização regional mais simples. O eixo político não foi tratado neste trabalho.

Por outro lado, a partir da ciência regional verifica-se uma preocupação na explicação de como as características econômicas de uma região afetam, positiva ou negativamente, às suas áreas circundantes, ou seja, seus impactos regionais. Nesse sentido, as contribuições provenientes das teorias do desenvolvimento econômico foram fundamentais para aprofundar esse entendimento, tanto as ortodoxas (preocupadas, principalmente, com os processos e variáveis macroeconômicos), 
quanto as mais heterodoxas (atentas aos padrões espaciais desiguais) (Scott e Storper, 2003).

A vertente heterodoxa, a mais esclarecedora, buscou as explicações dos impactos regionais, basicamente, por meio do papel central das aglomerações (cidades ou regiões). Estas, detentoras das condicionantes que promovem sobretudo o desenvolvimento econômico. Essa abordagem afirma que as externalidades positivas geradas nesses aglomerados, a exemplo dos fluxos de informação e aprendizagem e redes de negócios, possibilitam o desenvolvimento, ressaltando o papel fundamental das interdependências locacionais (Scott e Storper, 2003). Além do mais, muitas dessas externalidades espaciais podem estimular os processos de inovação tecnológica, tão importantes para o desenvolvimento (Audretsch, 2003).

A melhor versão da teoria heterodoxa é aquela relativa ao conceito de causação circular e cumulativa do espaço geográfico. Oriunda da teorização dos economistas Gunnar Myrdal e Albert O. Hirschman, esse conceito originou importantes aportes analíticos, como o dos polos de crescimento, também conhecidos como centros de crescimento regional (Scott e Storper, 2003). A causação está baseada na relação entre os diversos elementos ou "causas" (firmas, mão de obra especializada, etc.) que provocam o desenvolvimento de uma região econômica, na qual enfatiza-se que uma alteração de um elemento pode acarretar modificações nos outros. Ademais, conforme esta teoria regional, o desenvolvimento de uma região pode ter diferentes impactos no comportamento das regiões vizinhas, tanto positivos (efeito spread) quanto negativos (efeito backwash) (Scott e Storper, 2003).

Ambas considerações anteriores, sobre o desenvolvimento das cidades e o conceito de causação, fundamentam a construção da metodologia que se verá a seguir. Em outras palavras, um conjunto de métodos para explicitar o comportamento de uma economia urbana, metropolitana ou regional - oriunda dos quatro eixos do desenvolvimento das cidades - e os seus impactos regionais - proveniente da causação circular e cumulativa.

\section{Método e dados}

Este estudo se divide em duas partes complementares. A primeira consiste na caracterização da economia metropolitana de Portugal, portanto, com especial atenção aos maiores centros urbanos do país - Lisboa, Porto e Coimbra. Com base na literatura especializada no campo do ordenamento territorial, se constrói um quadro explicativo da estrutura econômico-urbana do país, salientando alguns dos aspectos alinhavados na seção anterior, ou seja, os eixos do desenvolvimento das cidades (institucional e das interações sociais). Assim, a análise incidirá nas principais relações econômicas que conformam o território português.

A segunda parte utiliza a análise exploratória de dados espaciais (AEDE) para mostrar a existência de impactos regionais no território, ou seja, se determinadas 
regiões têm influência sobre o desenvolvimento das vizinhas (Anselin, 1995; Sabater, Tur e Azorín, 2011; Griffith, 2005; Tartaruga, 2015). A AEDE procura verificar a existência de dependência espacial de dados. Hipótese que as distribuições de elementos no espaço geográfico seguem um padrão de inter-relação entre eles, os elementos mais próximos territorialmente, estão mais relacionados que os mais distantes. Observada frequentemente em variáveis naturais, esse tipo de dependência já é menos comum em variáveis humanas, como por exemplo, dados econômicos e demográficos (Buzai e Baxendale, 2006). Por essa razão, deve-se sempre verificar a dependência espacial de dados socioeconômicos.

A dependência espacial é analisada pela autocorrelação espacial de uma variável distribuída em diferentes localizações, no caso deste trabalho, regiões e municipalidades. Este tipo de análise, denominada univariada, mede o grau de relacionamento da variável de uma determinada unidade espacial com a mesma variável das unidades vizinhas. A análise de autocorrelação, uma das técnicas estatísticas de AEDE, tem por objetivo compreender as relações espaciais de uma distribuição de variáveis quando não se têm expectativas definidas sobre o comportamento do fenômeno (Sabater, Tur e Azorín, 2011). Por conseguinte, ver se a localização das variáveis tem influência sobre a distribuição espacial do fenômeno. A partir desta análise podem-se encontrar as seguintes situações:

- autocorrelação espacial positiva: existe semelhança entre os valores das unidades espaciais vizinhas, o que pode indicar uma tendência de aglomeração;

- autocorrelação espacial negativa: apresentam-se valores não semelhantes, nos quais, os valores das unidades vicinais são distintos da unidade em análise;

- inexistência de autocorrelação espacial: não ocorrência das situações anteriores, os valores das unidades contíguas se produzem de forma aleatória.

Retornando ao principal objetivo deste estudo -o impacto regional das regiões econômicas-, a variável escolhida para verificar a autocorrelação espacial foi a produtividade das unidades municipais, seguindo a metodologia de estudo anterior (Moreno Serrano e Vayá Valcarce, 2002). Para isso, analisaram-se os 278 concelhos (municipalidades) de Portugal continental, pois as unidades não continentais das Regiões Autonômas dos Açores e da Madeira não fariam sentido na análise espacial. A produtividade foi composta pela razão das seguintes variáveis anuais (Instituto Nacional de Estatística, 2019):

- valor acrescentado bruto (VAB) em euros, das empresas por concelho; e - pessoal ocupado (números absolutos) por concelho. ${ }^{1}$

1 A terminologia utilizada na instituição portuguesa para este dado é "Pessoal ao serviço das Empresas". Corresponde a todas as pessoas que atuaram em empresas ou instituições com contrato de trabalho formal ou sem (propriétários-gerentes, familiares não-remunerados,...) (INE, 2019). E as empresas são todas aquelas unidades de produção de bens e/ou serviços de todos os setores econômicos (agropecuária, indústria e serviços). 
Estas informações estatísticas estão distribuídas nas Unidades Territoriais para Fins Estatísticos (da sigla em francês NUTS), ano-base 2013, da União Europeia. Os anos em análise foram os de 2008 e 2017, respectivamente, o primeiro e o último da série histórica disponível.

A escolha da variável produtividade se justifica pela sua considerável relação com o desenvolvimento econômico e, consequentemente, com processos de inovação. Embora não seja uma ligação intrinsecamente direta, deve-se reconhecer que os países e regiões mais inovadores, de maneira geral, são aqueles que apresentam uma maior produtividade em comparação aos menos inovadores (Fagerberg, 2005).

Para a análise espacial foi construída uma matriz de proximidade da distribuição da produtividade com os seguintes parâmetros:

- critério de contiguidade: queen (considera todos os concelhos contíguos) e

- ordem de contiguidade: 2 (considera todos os vizinhos e os vizinhos a estes últimos vizinhos dos vizinhos).

Para averiguar a autocorrelação espacial foi utilizada a estatística I de Moran, tanto na sua versão global como local. ${ }^{2} \mathrm{~A}$ formulação global tem como alvo medir o comportamento geral de uma região subdividida em um conjunto de unidades e o índice correspondente varia de $-1 \mathrm{a}+1$, em que valores próximos $\mathrm{a}+1$ indicam autocorrelação espacial positiva, próximos a-1, autocorrelação negativa, e próximos a zero significam inexistência de autocorrelação ou padrão espacial.

A versão local da estatística visa mostrar onde se localizam os padrões espaciais dentro da área em estudo. Assim, as unidades espaciais são definidas em uma das seguintes quatro classes:

- alto-alto (high-high): autocorrelação positiva, valores da variável da unidade e dos vizinhos, em média, são altos (o conjunto de áreas contíguas deste tipo é denominado de hot spot);

- baixo-baixo (low-low): autocorrelação positiva, valores da variável da unidade e dos vizinhos, em média, são baixos (o conjunto destas áreas é conhecido como cold spots);

- alto-baixo (high-low): autocorrelação negativa, valor da unidade é alto, porém, os dos seus vizinhos são, em média, baixos; e

- baixo-alto (low-high): autocorrelação negativa, valor da unidade é baixo, porém, os de seus vizinhos são, em média, altos.

Tal classificação possui propriedades importantes para a análise regional que se quer realizar aqui. Com efeito, duas classes são pertinentes para verificar a causação circular e cumulativa. As regiões classificadas como "alto-alto" (formando hot spots) são aquelas que, provavelmente, possuem efeitos benéficos sobre os territórios vizinhos (efeito spread). Já as regiões "alto-baixo" indicam as que têm impactos

2 O programa computacional utilizado foi o GeoDa (Anselin, 2019). 
negativos nos espaços próximos (efeito backwash). Importante destacar que a conexão entre esta teoria regional e este método quantitativo é, aparentemente, inédita.

A estatística I de Moran também proporciona o estabelecimento do nível de significância do procedimento, o que aponta para a probabilidade de rejeição da hipótese nula (ausência de autocorrelação espacial). No caso da hipótese nula, a distribuição dos dados no espaço é aleatória, sem nenhum padrão espacial. Enquanto a ocorrência da hipótese alternativa indica uma autocorrelação positiva ou negativa. O estudo efetuado neste trabalho teve a seguinte orientação:

- nível de significância: $5 \%(0,05)$, portanto, uma confiança de $95 \%$.

Dessa forma, pode-se determinar as unidades espaciais que possuem uma autocorrelação significativa e, assim, uma dinâmica espacial particular merecedora de análise mais aprofundada.

\section{Resultados da análise empírica}

\subsection{Caracterização regional}

O perfil socioeconômico de Portugal, no contexto mundial, é de uma nação desenvolvida mediana pertencente à União Europeia (UE). Apesar dos eventuais problemas econômicos desse vínculo que, na sua maioria, foram de exclusiva responsabilidade de Portugal (Teixeira, 2017), a participação do país na UE traz oportunidades interessantes de desenvolvimento em parceria com os outros países europeus, sobretudo, no campo tecnológico. No contexto da UE, apresentam-se vantagens em inovações tecnológicas verdes (Perez e Leach, 2018; Mazzucato e Pérez, 2014), como aconteceu na Europa com inovações em outras áreas, pelo menos nos dois últimos séculos (Kaiser e Schot, 2014).

No que se refere a sua capacidade de inovação, em termos mundiais, Portugal está na posição número 32, em um total de 129 países (Global Innovation Index, 2019), corroborando sua posição mediana em desenvolvimento. No contexto europeu, Portugal é considerado, atualmente, um inovador moderado $\mathbf{3}$ conforme a última pesquisa, em 2018, sobre o desempenho em inovação dos países da UE (European Commission, 2019a), resultado que o posiciona em um lugar intermediário. Ressalta-se a importante ascensão do país em relação às edições anteriores dessa pesquisa que se inicia no ano de 2011.

Em termos regionais, o país mostra desempenhos destacáveis em algumas de suas regiões, nomeadamente, seus três principais centros urbanos: Lisboa, Porto e

3 Tal classificação de países possui quatro categorias que vão do mais inovador ao menos na seguinte ordem: líderes em inovação, inovadores fortes, inovadores moderados e inovadores modestos. 
Coimbra. Em outra pesquisa promovida pela Comissão Europeia, esses centros, no relatório correspondendo, respectivamente, a Área Metropolitana de Lisboa (AML), região Norte, onde está inserida a Área Metropolitana do Porto (AMP), e a região Centro, que incluía Região de Coimbra (RC), são considerados "inovadores fortes" (European Commission, 2019b). ${ }^{4} \mathrm{O}$ restante das regiões (Algarve, Alentejo e Regiões Autonômas dos Açores e da Madeira) são definidas como inovadores moderados.

$\mathrm{Na}$ mesma linha das considerações anteriores, estudos mais aprofundados sobre a organização econômica territorial portuguesa apontam alguns aspectos relevantes para a discussão à luz dos eixos de desenvolvimento das cidades, principalmente, nos temas relativos ao contexto econômico e das interações sociais no campo dos processos de inovação.

Efetivamente, a AML, a AMP e a RC (respectivamente, em Fundação Calouste Gulbenkian, 2015, 2014, 2016) apresentam um conjunto de infraestruturas de transporte, de comunicação, para organização urbana, de conectividade internacional, industriais, de serviços, agrícolas, universitárias e de inovação, de modo geral bem estruturadas, mas com algumas deficiências. Em função dessas estruturas, as três regiões são consideradas os principais motores de desenvolvimento do país, porém em graus diferentes de potência econômica e política, em uma hierarquia, em que, Lisboa possui indiscutível liderança, seguida de perto pelo Porto, e ultimada por Coimbra em uma posição bem mais modesta.

No domínio das interações socioeconômicas, particularmente, aquelas ligadas à inovação, também são as três metrópoles portuguesas que se destacam. Verificou-se que a maioria das colaborações e parcerias, em projetos de pesquisa e desenvolvimento (P\&D) financiados pelo governo português, $\mathbf{5}$ aconteceram entre universidades, centros de pesquisa e empresas localizadas nessas metrópoles (Gama, Barros e Fernandes, 2018). Ademais, por meio da análise dos projetos de P\&D da política do Horizonte 2020 da UE, na linha da especialização inteligente, ${ }^{6}$ constatou-se a consolidação de três importantes ecossistemas de inovação relacionados, respectivamente, aos três espaços metropolitanos (Santos, Marques, Ribeiro e Torres, 2018).

Entretanto, estes estudos abordam de forma sucinta os impactos regionais das três metrópoles sobre seus entornos. Esse é o objetivo da seguinte seção que enfrenta esta problemática através da análise espacial da produtividade.

4 Essa classificação apresenta 12 tipos, do mais ao menos inovador: líder +, líder, líder -, forte +, forte, forte -, moderado +, moderado, moderado -, modesto + , modesto e modesto -.

5 Por meio da Fundação para a Ciência e a Tecnologia (FCT), o órgão público nacional de apoio à pesquisa em ciência, tecnologia e inovação.

6 Política comunitária europeia de desenvolvimento econômico e tecnológico que segue a especialização inteligente, abordagem que, através da associação entre ciência e política pública, busca orientar os investimentos em pesquisa e inovação para promover transformações econômicas regionais (Gianelle, Kyriakou, Cohen e Przeor, 2016). 


\subsection{Análise espacial exploratória da produtividade}

Por sua vez, complementando a caracterização regional anterior, a análise exploratória de dados espaciais (AEDE) procura constatar se os principais centros urbanos portugueses irradiam impactos, benéficos ou não, sobre sua vizinhança. Para isso, utiliza-se como variável de análise a produtividade dos concelhos (municipalidades), mensurada a partir do VAB por pessoal ocupado, nos anos de 2008 e 2017.

A distribuição da produtividade no território lusitano mostra um comportamento, de modo geral, muito diferenciado, porém, com algumas particularidades regionais (Figura 1). Uma destas particularidades, tanto para 2008 como 2017, diz respeito à concentração dos valores mais altos de produtividade no litoral, sobretudo na faixa que vai da Área Metropolitana de Lisboa (AML) até a Área Metropolitana do Porto (AMP), incluindo, no meio dessa faixa, a Região de Coimbra (RC). Além disso, pode-se notar uma ligeira tendência a uma maior distribuição desta variável no período mais recente, em especial na metade sul.

Figura 1. Distribuição espacial da produtividade em Portugal continental - 2008 e 2017

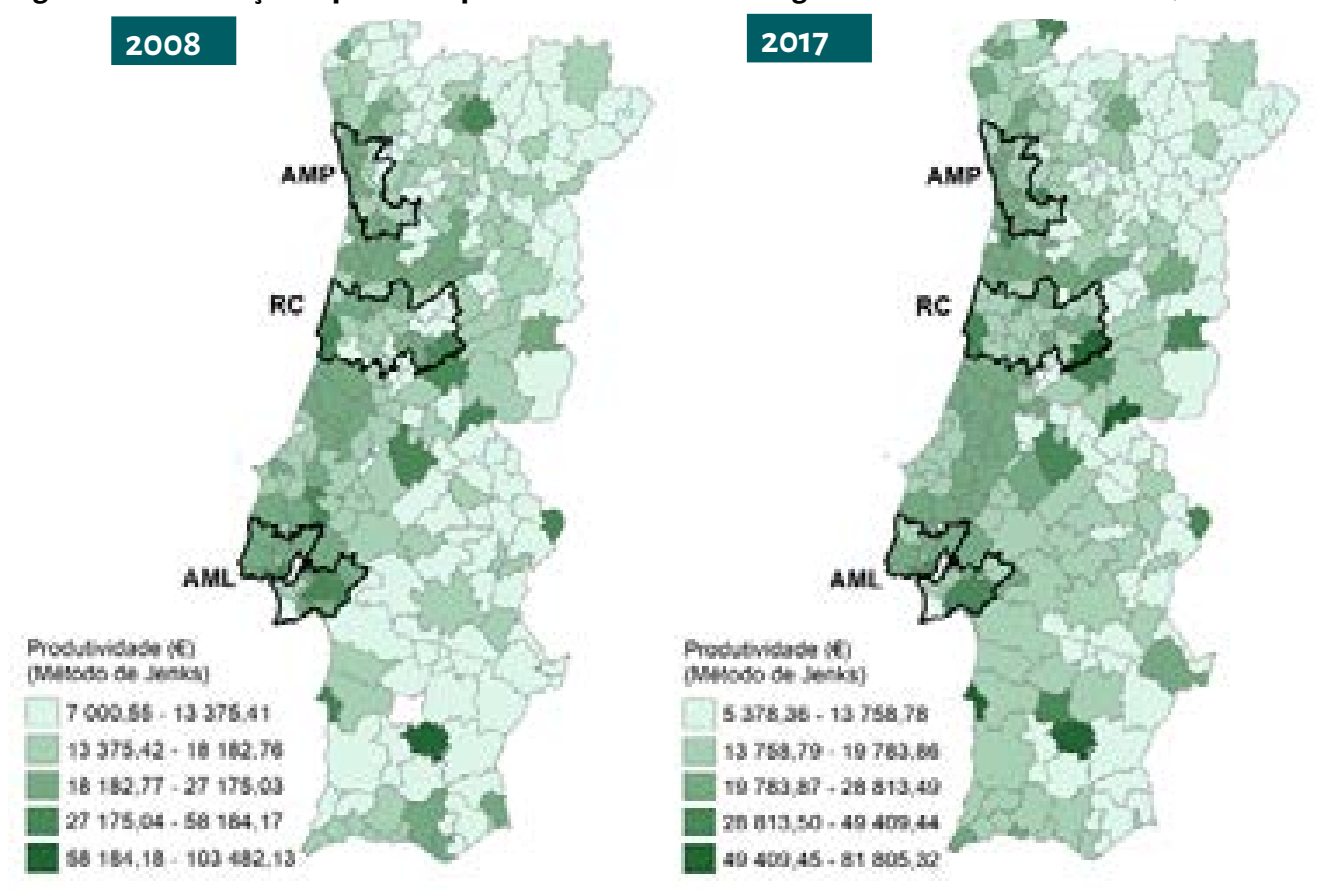

Nota: (a) AML (Área Metropolitana de Lisboa), AMP (Área Metropolitana do Porto) e RC (Região de Coimbra). (b) A classificação dos dados foi realizada através do método de Jenks, gerando um conjunto de classes de forma a maximizar a variância interclasses (maior diferenciação entre classes) e a minimizar a variância intraclasses (maior homogeneidade dentro de cada classe), portanto, uma classificação ótima. Fonte: Elaboração própria. 
Para a análise regional, entretanto, a simples análise visual não é a forma adequada para definir padrões de densidade de dados espaciais, em razão do problema da subjetividade do observador. Assim, os métodos de estatística espacial, como o I de Moran, são utilizados para estes fins, pois permitem encontrar padrões espaciais em termos de significância estatística (Pogodzinski e Kos, 2013).

A AEDE da variável de produtividade mostra que, apesar de não serem grandes, há mudanças significativas no padrão espacial entre 2008 e 2017 (Figura 2). Em geral, verificou-se o crescimento do número de concelhos em cada classe de padrão espacial, com exceção do "alto-baixo" (de 8 para 6 concelhos). Esta última informação é positiva, em termos de desenvolvimento, porque significa a diminuição de regiões com efeito backwash. Portanto, áreas mais desenvolvidas rodeadas de unidades pouco desenvolvidas, poderiam ser uma influência negativa daquelas regiões sobre estas. Vale destacar, evidentemente, que os concelhos de tipo "alto-baixo", geralmente, estão cercados pelo tipo "baixo-baixo" (sobretudo em 2017), este com o maior número de concelhos em ambos momentos. Ademais, estes últimos sofreram um aumento considerável no período (de 38 para 59 concelhos). Efetivamente, este resultado prejudicial revela que há uma forte relação entre concelhos com baixa produtividade (formando cold spots).

Figura 2. Análise exploratória espacial da produtividade em Portugal continental-2008 e 2017.

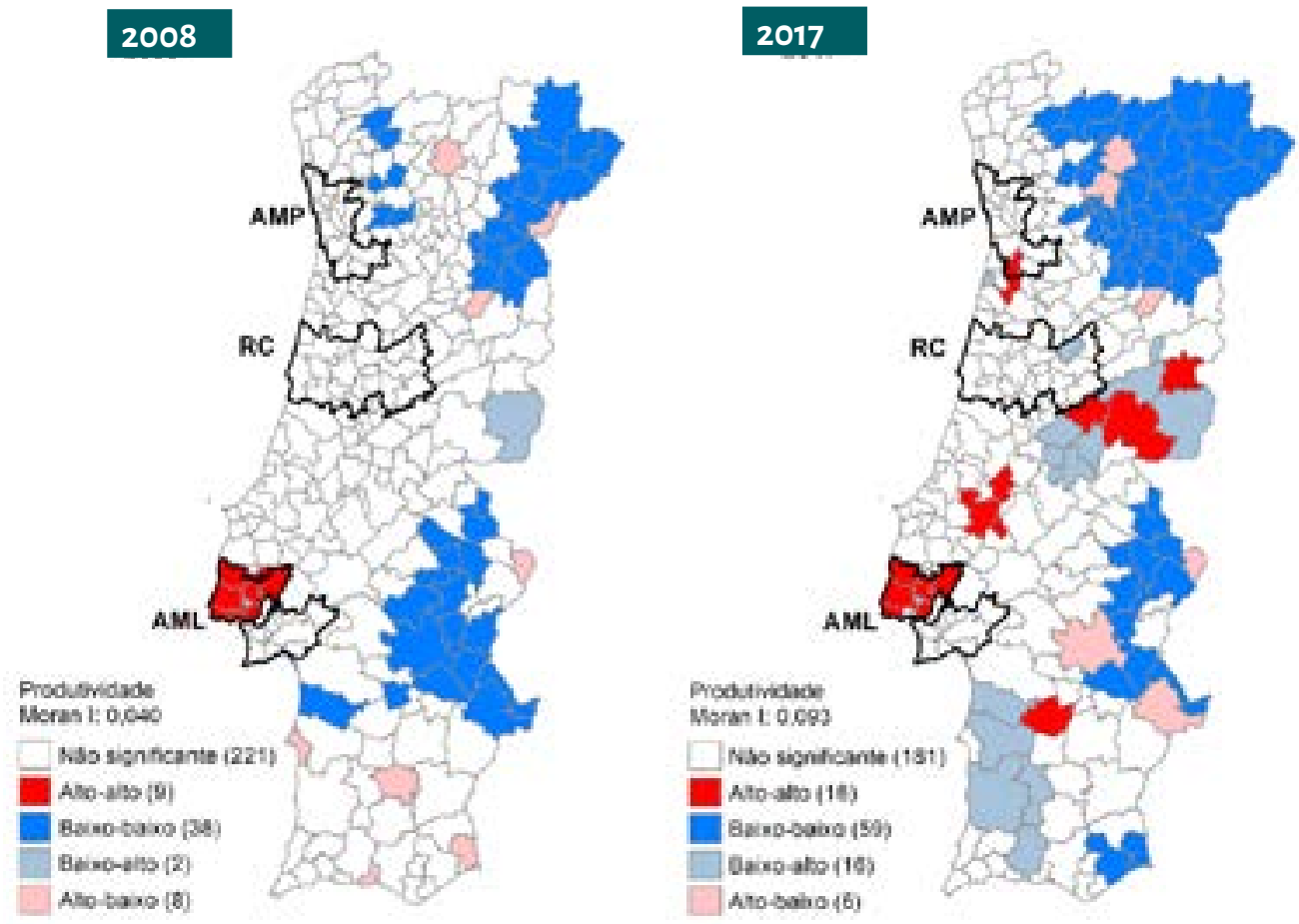

Nota: AML (Área Metropolitana de Lisboa), AMP (Área Metropolitana do Porto) e RC (Região de Coimbra). Fonte: Elaboração própria. 
O aumento da quantidade de concelhos com padrões espaciais significativos os não significantes reduziram de 221 para 181, entre 2008 e 2017 - reflete-se no crescimento do índice global de autocorrelação no período (Figura 2). O coeficiente que era de 0.040 passou a 0.093 , mostrando, em ambos períodos, a existência de autocorrelação espacial positiva. O que significa que há uma tendência geral de aglomeração em Portugal em relação a produtividade. Em outras palavras, a vizinhança importa para essa variável econômica.

A outra categoria espacial que importa nesta análise é a "alto-alto", ou seja, daqueles concelhos desenvolvidos que, possivelmente, têm efeito positivo sobre os vizinhos (efeito spread). Nesse período tiveram um aumento de 9 para 16 concelhos, o que é positivo em termos econômicos (Figura 2). Destaca-se que praticamente todos os concelhos que eram "alto-alto" em 2008, continuaram nesta mesma classe em 2017, significando uma expansão absoluta da classe.

Em relação à economia metropolitana portuguesa, pouco se pode dizer sobre o ano de 2008 (Figura 2). Com efeito, a AMP e a RC não apresentaram um único concelho com padrão espacial significativo. Contudo, na AML pode-se ver um conjunto de concelhos do tipo "alto-alto" (hot spot), com a exceção de um, no interior desta área metropolitana.

Em 2017, pode-se encontrar um comportamento espacial mais substancial relacionado a estas metrópoles, apesar de ainda escasso (Figura 2). Na área de Lisboa encontra-se, basicamente, o mesmo aglomerado de concelhos "alto-alto" (hot spot) do período anterior, agora somente os que estão no interior dos limites desta metrópole, totalizando sete concelhos onde está o de Lisboa. Na parte sul do Porto encontram-se dois concelhos, um dentro da área metropolitana e o outro fora, junto ao limite da metrópole. Em suma, um pequeno foco de efeito spread. E no caso de Coimbra, na sua porção sudeste, observam-se dois concelhos "alto-alto" externos aos limites da metrópole, denotando outro pequeno foco positivo.

Em suma, a AEDE dos espaços metropolitanos de Portugal demostrou que, em primeiro lugar, houve pouca alteração no padrão espacial no período analisado. Contudo, deve-se ressaltar que o intervalo de apenas nove anos, entre os dois momentos examinados, é um fator limitante intrínseco do estudo, pois muitos fenômenos sociais e econômicos sofrem modificações em hiatos de tempo maiores. Em segundo lugar, nas aglomerações do Porto e de Coimbra há pouca evidência estatística de que essas regiões tenham impactos regionais significativos, uma vez que o índice local de tais impactos não pode ser corroborado. Contrariamente, o índice global aponta para a possibilidade de haver algum impacto. Isso pode indicar a falta de estruturação territorial das relações econômicas nesses espaços. Terceiro, na principal metrópole portuguesa, Lisboa, pode-se verificar uma aglomeração autocorrelacionada positivamente dentro de sua área metropolitana, sem relacionamentos no seu exterior adjacente. Apesar de favorável, esta conclusão mostra a 
limitação das relações regionais - em termos econômicos - da principal economia metropolitana do país.

\section{Considerações finais}

Este artigo buscou analisar as transformações ocorridas no período recente nos espaços metropolitanos de Portugal e seus impactos regionais. As Áreas Metropolitanas de Lisboa e do Porto, e a Região de Coimbra, as principais metrópoles, têm influenciado economicamente suas regiões vizinhas. E, na existência desses impactos, estes são benéficos ou prejudiciais ao desempenho dos espaços da vizinhança. Não somente em Portugal, como em toda a Europa e em várias partes do mundo, essa última ocorrência, dos impactos negativos, aponta para um problema atual e em franco de crescimento que é o das desigualdades regionais (Rodríguez-Pose, 2018; lammarino, Rodriguez-Pose e Storper, 2019). Onde, nas regiões desfavorecidas, como pequenas cidades e áreas rurais, há um crescimento econômico cada vez menor frente a regiões "ganhadoras", sobretudo nos grandes aglomerados urbanos. Isso acarreta diversos problemas, como o crescimento do populismo (Rodríguez-Pose, 2018), que também tendem a minar o crescimento das regiões mais desenvolvidas.

Para analisar os impactos regionais das metrópoles portuguesas, foi utilizado um quadro teórico e metodológico que une os campos científicos da geografia econômica e da estatística espacial. Teoricamente, a abordagem dos eixos - economia, instituições, interações sociais e política - de desenvolvimento das cidades (Storper, 2013) e do conceito de causação circular e cumulativa do espaço geográfico (Scott e Storper, 2003) foram essenciais para fundamentar os métodos quantitativos usados. Quanto à metodologia, aplicou-se a estatística I de Moran que é uma técnica de análise exploratória de dados espaciais (Anselin, 1995), que serve para verificar a existência de relação espacial entre unidades territoriais vizinhas. Para isso, a produtividade das empresas portuguesas, nos anos de 2008 e 2017, foi a variável principal da análise espacial.

Os resultados da análise espacial mostram um cenário territorial diversificado e pouco estruturado. Primeiro, o conjunto do território português apresentou, em ambos os períodos, uma autocorrelação global significativa. De modo geral, há uma tendência de influência ou relação espacial entre os concelhos (municipalidades). Segundo, ainda no quadro territorial geral, percebe-se conjuntos importantes de aglomerações de concelhos com baixo desempenho econômico (cold spots), localizados no interior do país e fora do espectro das principais metrópoles. Essa constatação ressalta o processo de decrescimento econômico e de despovoação que vem assolando o país nos últimos anos. Terceiro, na Área do Porto, no norte do país, não se pôde determinar relacionamentos espaciais, tanto positivos como negativos, significativos. Da mesma forma, a Região de Coimbra não apresentou relações 
significativas. Tais resultados fornecem indícios da ausência de estruturação econômica dessas regiões nos seus interiores e nos entornos próximos, o que é prejudicial para uma consolidação do desenvolvimento econômico em geral. Quarto, distinta às regiões do Porto e de Coimbra, a Área Metropolitano de Lisboa revelou uma importante aglomeração de concelhos de desempenho positivo (hot spot) no interior desta região, incluindo a concelho de Lisboa. Entretanto, não se verificaram relacionamentos espaciais (impactos) com as áreas adjacentes a esta metrópole.

Portanto, em Portugal é evidente a concentração dos processos de desenvolvimento econômico na metrópole lisboeta, e, basicamente, inconclusiva nas do Porto e Coimbra. Tal conclusão está em linha com outros estudos que enfatizam essa concentração em termos gerais (Rodríguez-Pose e Crescenzi, 2008; Morgan, 2004). Isso se explica por diversos fatores com clara tendência de agregação na atividade econômica, como as inovações, as especializações, o capital social e os contatos face a face para o transbordamento de conhecimentos. Outra explicação para o baixo grau dos relacionamentos espaciais dentro do território português é a forte influência das ligações comerciais internacionais, processos estes vinculados com a globalização econômica. Nesse sentido, outros estudos mostram essa inclinação ao exterior, como no campo dos projetos de investigação e desenvolvimento, realizados no país, com um componente crescente em parcerias com instituições internacionais (Gama et al., 2018). Ou, também, como no caso especial de Lisboa em sua propensão aos vínculos científicos e econômicos com o exterior (Fundação Calouste Gulbenkian, 2015).

Cabe ressaltar, por fim, que embora o país possua importantes centros econômicos, como Lisboa, Porto e Coimbra, essa estrutura regional peca pelo pouco relacionamento com seus entornos regionais. Apesar de apresentar importantes ligações com o exterior, o que é louvável, essa estrutura perde parte de sua capacidade de articulação regional. Como provam as experiências mais bem-sucedidas de desenvolvimento socioeconômico, deve-se buscar a combinação entre articulação local/ regional com ligações internacionais, em que ambas acabam apoiando-se reciprocamente (Bathelt et al., 2004). Com efeito, a maior organização local alarga a competência regional de absorção dos fluxos externos, especialmente de conhecimentos. Dessa forma, pode-se dizer que Portugal possui quase todas as ferramentas, considerando o papel de suas metrópoles, para um desenvolvimento mais consistente.

\section{Agradecimentos}

Agradecemos a leitura atenta de este texto de Fernanda Queiroz Sperotto, assim como as sugestões e críticas dos dois pareceristas deste artigo. 


\section{Referências}

ANSELIN, L. (1995). Local Indicators of Spatial Association-LISA. Geographical Analysis, 27(2), 93-115.

ANSELIN, L. (2019). Ceoda (1.14.0) [Computer software]. Recuperado de https:// spatial.uchicago.edu/geoda

AUDRETSCH, D. B. (2003). Innovation and Spatial Externalities. International Regional Science Review, 26(2), 167-174. Recuperado de https://journals.sagepub.com/doi/ pdf/10.1177/0160017602250973

BATHELT, H., \& GLÜCKLER, ]. (2014). Institutional change in economic geography. Progress in Human Geography, 38(3), 340-363.

BATHELT, H., MALMBERG, A., \& MASKELL, P. (2004). Clusters and knowledge: local buzz, global pipelines and the process of knowledge creation. Progress in Human Geography, 28(1), 31-56.

BUZAI, G. D., \& BAXENDALE, C. A. (2006). Análise socioespacial con Sistema de Información Geográfica. Buenos Aires, Argentina: Lugar Editorial.

EUROPEAN COMMISSION. (2019a). European Innovation Scoreboard. Recuperado de https://ec.europa.eu/growth/industry/innovation/facts-figures/scoreboards_en

EUROPEAN COMMISSION. (2019b). Regional Innovation Scoreboard. Recuperado de https://ec.europa.eu/growth/industry/innovation/facts-figures/regional_en

FACERBERG, ]. (2005). Innovation: a guide to the literature. In ]. Fagerberg, D. C. Mowery \& R. R. Nelson (Eds.), The Oxford Handbook of Innovation (pp. 1-26). Oxford, UK: Oxford University Press. Recuperado de http://fds.oup.com/www. oup.co.uk/pdf/0-19-926455-4.pdf

FUNDAÇÃO CALOUSTE GULBENKIAN. (2014). Noroeste Global. Lisboa, Portugal: Fundação Calouste Gulbenkian. Recuperado de https://gulbenkian.pt/ programas/gulbenkian-cidades/noroeste-global/

FUNDAÇÃO CALOUSTE GULBENKIAN. (2016). Portugal no Centro. Lisboa, Portugal: Fundação Calouste Gulbenkian. Recuperado de https://gulbenkian.pt/ programas/gulbenkian-cidades/portugal-no-centro/

FUNDAÇÃO CALOUSTE CULBENKIAN. (2015). Uma Metrópole para o Atlântico. Lisboa, Portugal: Fundação Calouste Culbenkian. Recuperado de https://gulbenkian.pt/ programas/gulbenkian-cidades/arco-metropolitano-lisboa/

GAMA, R., BARROS, C., \& FERNANDES, R. (2018). Science Policy, R\&D and Knowledge in Portugal: an Application of Social Network Analysis. Journal of the Knowledge Economy, 9(2), 329-358. doi: https://doi.org/10.1007/s13132-017-0447-3

GIANELLE, C., KYRIAKOU, D., COHEN, C., \& PRZEOR, M. (Eds.). (2016). Implementing Smart Specialisation Strategies: a handbook. Brussels, Belgium: European Commission. Recuperado de http://s3platform.jrc.ec.europa.eu/-/draftimplementing-smart-specialisation-strategies-a-handbook 
GLOBAL INNOVATION INDEX. (2019). Recuperado de https://www. globalinnovationindex.org/gii-2019-report

GRIFFITH, D. A. (2005). Spatial Autocorrelation. In K. Kempf-Leonard (Ed.), Encyclopedia of Social Measurement. Volume 3 (pp. 581-590). Amsterdam, Netherlands: Elsevier.

IAMMARINO, S., RODRICUEZ-POSE, A., \&STORPER, M. (2019). Regional inequality in Europe: evidence, theory and policy implications. Journal of Economic Geography, 19(2), 273-298. doi: https://doi.org/10.1093/jeg/lbyo21

INSTITUTO NACIONAL DE ESTATÍSTICA. (2019). Sistema de contas integradas das empresas. Recuperado de https://www.ine.pt/xportal/ xmain?xpid=INE\&xpgid=ine_base_dados\&contexto=bd\&selTab=tab2

KAISER, W., \& SCHOT, J. (2014). Writing the rules for Europe: Experts, cartels, and international organizations. London, UK: Palgrave Macmillan.

MAZZUCATO, M., \& PÉREZ, C. (2014). Innovation as growth policy: the challenge for Europe. SPRU Working Paper Series, 2014(13), 1-28. Recuperado de https://www. sussex.ac.uk/webteam/gateway/file.php?name=2014-13-swps-mazzucato-perez . pdf\&site $=25$

MORENO SERRANO, R., \& VAYÁ VALCARCE, E. (2002). Econometría espacial: nuevas técnicas para el análisis regional. Una aplicación a las regiones europeas. Investigaciones Regionales, 1, p. 83-106. Recuperado de http://www.aecr.org/ images/ImatgesArticles/2007/04-Moreno.pdf

MORGAN, K. (2004). The exaggerated death of geography: learning, proximity and territorial innovation systems. Journal of Economic Geography, 4(1), 3-21.

PEREZ, C., \& LEACH, T. M. (2018). A Smart Green 'European Way of Life': the Path for Growth, Jobs and Wellbeing. Beyond the Technological Revolution Working Paper Series, 2018(1), 3-24. Recuperado de http://beyondthetechrevolution.com/wpcontent/uploads/2014/10/BTTR_WP_2018-1.pdf

POCODZINSKI, J. M., \& KOS, R. M. (2013). Economic development and GIS. Redlands, USA: Esri Press.

RODRÍCUEZ-POSE, A. (2018). The revenge of the places that don't matter (and what to do about it). Cambridge Journal of Regions, Economy and Society, 11 (1), 189-209. RODRÍCUEZ-POSE, A., \& CRESCENZI, R. (2008). Mountains in a flat world: why proximity still matters for the location of economic activity. Cambridge Journal of Regions, Economy and Society, 1(3), 371-388. doi:10.1093/cjres/rsno11

RODRÍCUEZ-POSE, A., \&STORPER, M. (2006). Better Rules or StrongerCommunities? On the Social Foundations of Institutional Change and Its Economic Effects. Economic Geography, 82(1), 1-25.

SABATER, L. A., TUR, A. A., \& AZORÍN, J. M. N. (2011). Análise Exploratória de Dados Espaciais (AEDE). In ]. S. COSTA, T. P. DENTINHO \& P. NIJKAMP (Coords.), Compêndio de Economia Regional - Volume II: métodos e técnicas de análise regional 
(pp. 259-293). Cascais, Portugal: Princípia.

SANTOS, H., MARQUES, T. S., RIBEIRO, P., \& TORRES, M. (2018). Especialização inteligente: as redes de projetos europeus $\mathrm{H}_{2} \mathrm{O} 2 \mathrm{O}$ com ancoragem em Portugal. Livro de Atas das Jornadas de Ceografía Económica: La Ceografía de las Redes Económicas y la Ceografía Económica en Rede, 8, 13-35. Recuperado de http://ler. letras.up.pt/uploads/ficheiros/16320.pdf

SCOTT, A. J., \& STORPER, M. (2003). Regions, globalization, development. Regional Studies, 37(6\&7), 579-593. doi: https://doi.org/10.1177/0042098016634002

SCOTT, A. J., \& STORPER, M. (2015). The nature of cities: The Scope and Limits of Urban Theory. International Journal of Urban and Regional Research, 39(1), 1-15.

STORPER, M. (2010). Why Does a City Grow? Specialisation, Human Capital or Institutions? Urban Studies, 47(10), 2027-2050. doi: https://doi. org/10.1177/0042098009359957

STORPER, M. (2011). Justice, efficiency and economic geography: should places help one another to develop? European Urban and Regional Studies, 18(3), 3-21.

STORPER, M. (2013). Keys to the city: how economics, institutions, social interactions, and politics shape development. Princeton, U.S.A.: Princeton University Press.

STORPER, M. \& VENABLES, A. ]. (2004). Buzz: face-to-face contact and the urban economy. Journal of Economic Geography, 4(4), 351-370. doi: https://doi. org/10.1093/jnlecg/lbho27

TARTARUGA, I. G. P. (2015). Técnicas de análise de dependência espacial de dados socioeconômicos. In G. F. W. Radomsky, M. A. Conterato \& S. Schneider (Orgs.), Pesquisa em desenvolvimento rural: técnicas, base de dados e estatísticas aplicadas aos estudos rurais (pp. 245-261). Porto Alegre, Brasil: Editora da UFRGS.

TEIXEIRA, P. B. (2017). O Euro e o crescimento económico. Lisboa, Portugal: Fundação Francisco Manuel dos Santos. 